\title{
The continuous and changing impact of affect on risky decision-making
}

Erkin Asutay ( $\square$ erkin.asutay@liu.se )

Linköping University

Daniel Västfjäll

Linköping University

\section{Research Article}

Keywords: risky choice, affect, arousal, loss aversion, risk sensitivity, hierarchical Bayesian modeling

Posted Date: September 16th, 2021

DOI: https://doi.org/10.21203/rs.3.rs-889022/v1

License: (9) This work is licensed under a Creative Commons Attribution 4.0 International License. Read Full License 
The continuous and changing impact of affect on risky decision-making

\author{
Erkin Asutay ${ }^{1, *} \&$ Daniel Västfjäll ${ }^{1,2}$ \\ ${ }^{1}$ Department of Behavioral Sciences and Learning, Linköping University, Sweden \\ ${ }^{2}$ Decision Research, OR
}

* Correspondence concerning this article should be addressed to Erkin Asutay, Department of Behavioral Sciences and Learning, Linköping University, SE 58183 Sweden.

erkin.asutay@liu.se 


\begin{abstract}
Affective experience has an important role in decision-making with recent theories suggesting a modulatory role of affect in ongoing subjective value computations. However, it is unclear how varying expectations and uncertainty dynamically influence affective experience and how dynamic representation of affect modulates risky choices. Using hierarchical Bayesian modeling on data from a risky choice task $(\mathrm{N}=101)$, we find that the temporal integration of recently encountered choice parameters (expected value, uncertainty, and prediction errors) shapes affective experience and impacts subsequent choice behavior. Specifically, experienced arousal prior to choice was associated with increased loss aversion, risk aversion, and choice consistency. Taken together, these findings provide clear behavioral evidence for continuous affective modulation of subjective value computations during risky decision-making.
\end{abstract}

Keywords: risky choice, affect, arousal, loss aversion, risk sensitivity, hierarchical Bayesian modeling 


\section{Introduction}

Affect and emotions have a central role in judgment and decision-making. Previous studies have demonstrated affective modulation of decision-making under risk and uncertainty $[1,2]$. The recent theories suggest that affective processes modulate information processing and decision-making in a context dependent manner, wherein affective feelings are used as information by the decision-maker, influencing the ongoing subjective value computations $[1,3]$. However, despite an abundant literature on affect and decision-making, there is still a lack of understanding of the mechanisms for the continuous modulation of decision-making by affective experience. The relationship between affect and decisions is complex because affective experience not only influences perception of decision parameters and subjective value computations but also is dynamic and continuously influenced by the encountered information in a decision context. Here, using a risky decision-making task and hierarchical Bayesian modeling, we investigated how affective experience fluctuates as a function of varying choice variables and how trial-to-trial variations in affective experience influence risky decision-making in a dynamic context.

The two longstanding approaches to studying the role of affective processes in risky decision-making has been either to induce an affective state in a group of individuals and study their choices in an experimental paradigm (i.e., incidental affect), or to measure affective responses in a decision context and study the correlations between affective experience and choice (i.e., integral affect). When it comes to incidental affect, previous research has shown that incidental anger and fear were associated with a subsequent increase and decrease in risk taking, respectively [4]. However, the opposite effects of incidental anger and fear on risk taking were found depending on the decision context [5] or when the task involved estimating a social instead of a monetary risk [6]. Yet, others found that anger leads to more optimistic risk estimations only in men but not in women [7]. This pattern of results 
leaves us with open questions about the role of affective experience on risk taking. Moreover, integral and incidental affect can have a differential influence on risky decisions $[8,9]$. One of the first investigations on the role of integral affect in risky decision-making found that anticipatory physiological arousal indexed by skin conductance responses is associated with less risky choices [10]. Additionally, increased autonomic arousal was associated with decreased risk taking especially when probability of winning was small [11]. However, it is still unclear how affect continuously modulates risky choice in a dynamic context.

Two decision parameters, risk sensitivity and loss aversion, are often used to explain differences in risk preferences [12]. Risk sensitivity determines whether the decision indicates a risk-seeking or a risk-averse behavior, whereas loss aversion is the tendency to weigh potential losses more heavily than equivalent potential gains. Research has shown that higher arousal responses to losses relative to gains correlates with the degree of individual loss aversive behavior [13]. However, risk preferences are often unstable and context dependent [1]. Additionally, previous research has mainly focused on average risk preferences at the individual level, and not so much on the intraindividual variations in risky decision-making as a result of ongoing affective processing. Given the dynamic and continuous nature of affect, there is a need to adopt an experimental framework attempting to capture the key dynamic parameters of how moment-to-moment affective fluctuations modulate subjective value computations in risky choice.

Affect is characterized as a stream of fluctuations in an organism's neurophysiological state representing its ongoing relationship with the environment [14]. Hence, it is a dynamic and temporally dependent mental process. Previous research has shown that momentary affective experience is shaped by a temporal integration of the currently active information and previously experienced affect $[15,16]$. Furthermore, it has been shown that moment-tomoment happiness ratings during risky decision-making depends on the prediction error 
history [17]. However, the temporally dependent nature of affect is often not considered when studying its role in decision-making. In most studies, researchers use paradigms, in which individuals make a series of randomized, independent choices between different options. Then, the choice behavior is often modeled based on the given information and random noise [18], while affect being operationalized either as induced mood states that are assumed to be static and long-lasting or as stable individual difference measures. This is at odds with the temporally dependent and dynamic structure of mental processes like affect [19]. Hence, there is a need to model affect dynamics reflecting the temporal integration of previously encountered events to understand how moment-to-moment variations in affective experience influence risky decision-making. The purpose of the current study is to investigate (1) affective fluctuations as a function of varying expectations, uncertainty, and prediction errors and (2) the variation in risky decision-making as a result of ongoing affective experience. We used a risky decision-making task, in which individuals make a series of risky monetary decisions and report their momentary affective experience. We adopted a computational modeling approach to capture the key dynamic variables determining affective experience based on previously encountered choice variables (i.e., expected value, uncertainty, and prediction error). Moreover, we modeled the influence of trial-to-trial variations in affective experience on decision parameters of loss aversion, risk sensitivity, and choice consistency.

\section{Method}

\section{Participants}

We recruited 108 individuals through a university participant pool. The study was run online using Inqusit (Inqusit version 5) and participants were compensated after the study. Each participant received a 75 SEK (approx. \$7.5 at the time of the study) participation fee. In addition, one of the decisions for each participant was selected randomly and played out 
for real at the end of the study. The outcome of this gamble defined the final compensation $($ Mean $=80$ SEK, SD = 14 SEK $)$. Data from seven individuals were excluded from all analyses. One participant provided the same response in more than $80 \%$ of the trials. Six participants were removed because they provided affect ratings without moving the scale sliders in more than $80 \%$ of the trials. Hence, the final sample consisted of 101 individuals (34 females, Mean age $=24, \mathrm{SD}=5.2$ ). The study was conducted in accordance with the ethical standards in the Declaration of Helsinki and approved by the regional ethics committee in Linköping, Sweden. Formal power analysis was not conducted as we used Bayesian analyses. The data collection was open for two weeks after which we stopped the collection and analyzed the data. All data and modeling codes are publicly available at https://osf.io/ryfu9/. The modeling was done using Stan [20] and fitted in R using the rstan package [21]. The study design and analyses were not pre-registered.

\section{Risky decision-making task}

Participants viewed 50 monetary gambles each consisting of four possible outcomes (gains and/or losses) with associated probabilities. The possible outcomes of a given gamble are selected randomly from a normal distribution $($ Mean $=0$ SEK, $S D=18 \mathrm{SEK})$ and rounded to the nearest 5 SEK (e.g., $13.4 \sim 15$; and -7.9 -10). In addition, we apply the following limitations: (1) the maximum possible loss or gain will not exceed 75 SEK, (2) all four possible outcomes of a given gamble will be different, (3) ' 0 SEK' will not be a possible outcome, and (4) the expected value of any gamble will not exceed \pm 25 SEK. When generated in this way, expected values come from a normal distribution with 0 SEK mean and 10 SEK standard deviation (Figure S1 in Supplementary Information). The monetary gambles were generated separately for each participant.

The following independent choice variables were extracted from each gamble: expected value $(\mathrm{EV})$, uncertainty $(\mathrm{U})$, and prediction error $(\mathrm{PE})$. EV is determined as 


$$
E V=\sum_{i}^{4} x_{i} * p_{i}
$$

where $x_{i}$ is a monetary outcome and $p_{i}$ is its probability. Uncertainty (U) is given by the variance of the $\mathrm{EV}$.

$$
U=\sum_{i}^{4}\left(x_{i}-E V\right)^{2} * p_{i}
$$

$U$ was square-root transformed to reduce the skewness inherent in the variable, and centered around zero. PE is given as the difference between the actual outcome and EV.

$$
P E= \begin{cases}\text { Outcome }-E V, & \text { if gamble is accepted } \\ 0, & \text { if gamble is rejected }\end{cases}
$$

\section{Experimental procedure}

Each participant, after reading the instructions and giving informed consent online, went through the gambles. In each trial, participants viewed the given gamble and decided whether to accept or reject it (Figure 1), after which participants received feedback regarding the outcome of the trial. Participants viewed the outcome of the gamble if they chose to play it; otherwise, they viewed " $O S E K$ " as the outcome. Following the feedback, participants reported how they currently feel on visual analog scales of valence (unpleasant to pleasant) and arousal (sleepiness to high activation). Participants were explicitly instructed to assess how they currently feel at the time of reporting. Valence and arousal ratings were individually standardized. 


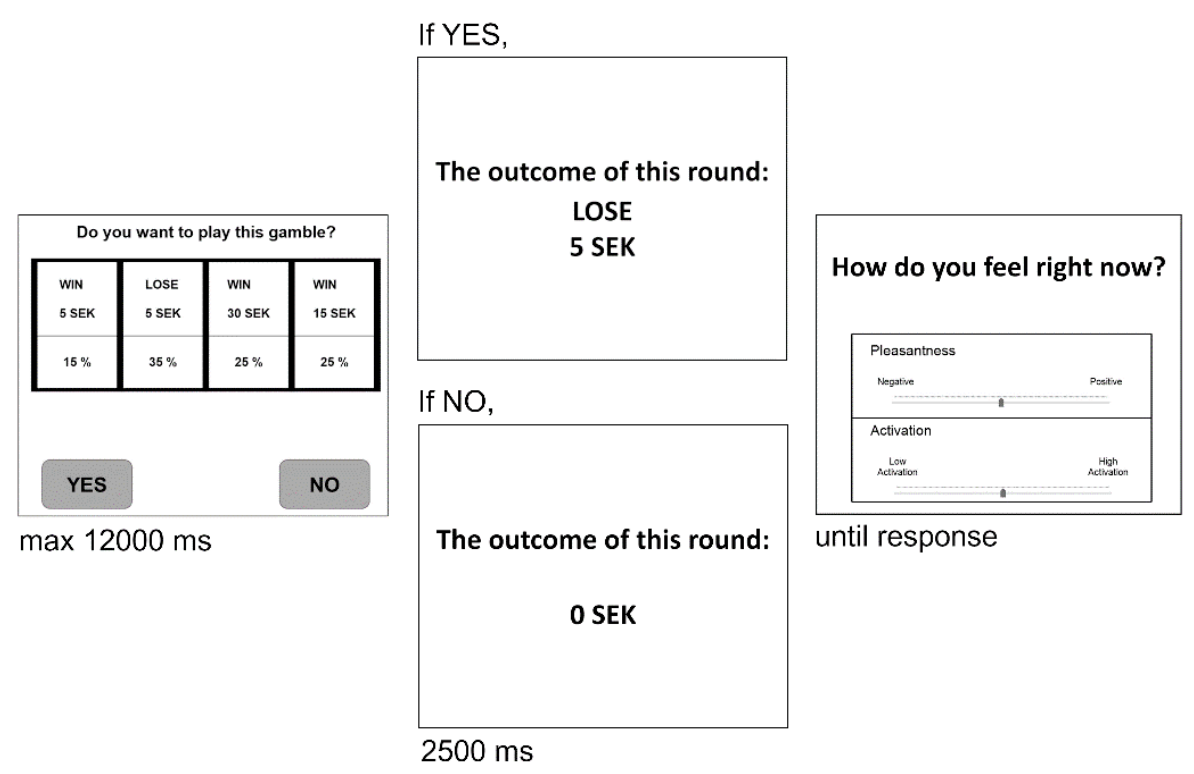

Figure 1. Trial structure. Participants viewed a gamble with four possible outcomes and decided whether to accept it or not, after which they received feedback. Following the feedback, they were asked to report how they felt using pleasantness and activation scales.

\section{Data analysis and computational modeling}

We used hierarchical Bayesian analysis (HBA) to study affective fluctuations as a function of varying decision variables and the potential influence of this changing affective experience on risky decision-making. HBA estimates posterior distributions that reflect uncertainty for parameters at group and individual levels and optimizes the tradeoff between random and fixed-effects models of individual differences [22-24]. Individual participants are constrained by group distributions, but they can also vary from the group distributions to the extent their data are diagnostic.

\section{Affective experience}

We have used a previously established model that generates a momentary subjective affective state based on the integration of previously encountered information $[17,25]$. Trial- 
by-trial affective experience is generated based on an exponential decay of the influences of previous events.

$$
A E_{t, i}=w_{0, i}+w_{E V, i} \sum_{j=1}^{t} \gamma_{i}^{t-j} * E V_{j, i}+w_{U, i} \sum_{j=1}^{t} \gamma_{i}^{t-j} * U_{j, i}+w_{P E, i} \sum_{j=1}^{t} \gamma_{i}^{t-j} * P E_{j, i}
$$

$A E_{t, i}$ is affective experience (valence and arousal) for individual $i$ at time point $t . E V_{j, i}$, $U_{j . i}$, and $P E_{j . i}$ are decision variables in trial $j$. The free parameters of the model in are $w$ and $\gamma$ terms determining the affective integration of encountered information. The $w_{0, i}$ is the constant term representing a baseline affective experience around which an individual fluctuates during the task. The parameters $w_{E V, i}, w_{U, i}$, and $w_{P E, i}$ are the weights on the decision variables $E V, U$, and $P E$, representing the degree to which these variables impact an individual's affective experience. Finally, $\gamma_{i}$ is an individual forgetting factor adjusting the influence of recent events in comparison to earlier events with $0 \leq \gamma \leq 1$. This parameter defines the relative impact of earlier vs. later stimuli on momentary affect. As $\gamma$ approaches to 1 , each trial is weighted evenly, while a $\gamma$ of 0 means that only the current trial determines the current affective experience.

We used HBA to estimate group and individual level parameters separately for valence and arousal ratings. The parameters for each individual were modeled to be distributed according to a normal (the weight parameters), beta (forgetting factors), or lognormal (individual variance) centered around a group mean with a group variance with weekly or non-informative priors (for details on priors and fitting procedure, see the Supplementary Information). The model was coded in Stan [20] and fitted in R using the rstan package [21]. Markov chain Monte Carlo (MCMC) sampling methods were used to estimate the posterior distributions of the parameters. We fit the model separately to valence and arousal ratings using 8 sampling chains for 3,000 iterations each, 1,000 of which were 
discarded as warm-up samples. This resulted in 16,000 samples for each parameter. Trace plots of the group level parameters were visually inspected to ensure convergence. We also ensured that all $\hat{R}$ values were under 1.01 which indicates that chains have adequate mixing [26]. Additionally, posterior predictive checks showed that the models captured the overall valence and arousal distributions (Figure 2).
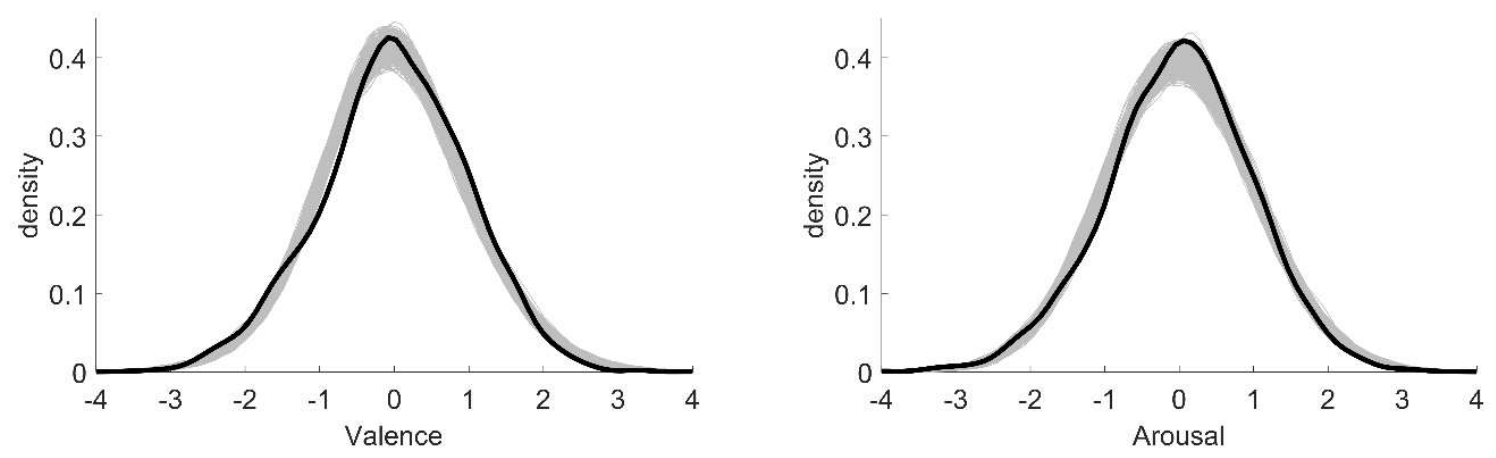

Figure 2. Posterior predictive checks for the affect model. Valence and arousal distributions are shown (solid black, smoothed with kernel density) together with posterior predictive simulations (1000 simulations) from the affective experience model (grey).

\section{Choice Behavior}

Base model. We used a choice model based on prospect theory to parametrize participants choice behavior based on subjective utility computations.

$$
\begin{aligned}
& u_{i}=x_{i}{ }^{\rho} \quad \text { if } x_{i}>0 \\
& u_{i}=-\lambda\left(-x_{i}\right)^{\rho} \quad \text { if } x_{i}<0 \\
& V_{\text {gamble }}=\sum_{i=1}^{4} u_{i} \cdot p_{i}
\end{aligned}
$$

Equation 5 shows the utility calculation of a possible monetary outcome, $x_{i}$, with two free parameters, $\lambda$ and $\rho . \lambda$ determines the asymmetric weighting of gains and losses and is 
also known as loss-aversion, a central tenet of prospect theory with $\lambda>1$ indicating loss aversive behavior [12]. $\rho$ reflects the curvature of the value function in prospect theory and reflects risk sensitivity. In the context of risky decision-making, $\rho<1$ indicates risk averse behavior, while $\rho>1$ indicates risk seeking behavior. $V_{\text {gamble }}$ in Equation 6 represents the subjective value of a gamble, which is the probability $\left(p_{i}\right)$ weighted sum of the utilities of the four possible outcomes. The probability of accepting the risky gamble then depends on $V_{\text {gamble. }}$

$$
p(\text { risk })=\frac{1}{1+e^{-c \cdot V_{\text {gamble }}}}
$$

Here, $c(0 \leq c \leq 20)$ is choice consistency (i.e., inverse temperature) parameter that captures randomness in participants choices, in which $c=0$ indicates fully random decisions.

Similar to the affect model, we used HBA to estimate group and individual level parameters. The parameters $(\lambda, \rho$, and $c)$ for each individual were modeled to be distributed around a group mean with a group variance (for details on priors and fitting procedure, see the Supplementary Information).

Trial-by-trial variations. To test our hypothesis that changing affective experience depending on varying decision variables dynamically influences risky decision-making, we implemented regression coefficients by reparametrizing the model parameters $(\lambda, \rho$, and $c)$ so that they were influenced by trial-by-trial variations of valence and arousal. We estimated posterior distributions for the degree to which loss aversion, risk sensitivity and choice sensitivity were altered by the variations in momentary affective experience. Below is a formulation of trial-by-trial affective fluctuations of the loss aversion parameter.

$$
\lambda_{i, t}=\lambda_{i}+\beta_{V} \cdot \operatorname{Valence}(t)+\beta_{A} \cdot \operatorname{Arousal}(t)
$$


The regression coefficients, $\beta_{V}$ and $\beta_{A}$, reflect the degree to which loss-aversion changes with experienced valence and arousal before the current decision (i.e., reported at the end of the previous trial). $\lambda_{i}$ in Equation 8 is the average loss aversion for participant $i$ and is drawn from the group-level distribution as in the base model above. $\lambda_{i, t}$ is the trial-by-trial loss aversion fluctuating around the individual average, and these fluctuations are determined by affective experience. We additionally implemented equivalent regressions for the risk sensitivity $(\rho)$ and the choice consistency $(c)$ parameters. We only estimated the group level posterior distributions for the regression coefficients, rather than assuming separate regression coefficients for each variable and participant, as this would substantially increase the number of nodes in the model (e.g., [27]).

Model fitting and comparison. We first fitted the base model without including affective experience data. Next, we tested whether trial-to-trial variations in affective experience influence decision parameters. We formulated a full model, in which self-reported valence and arousal before a trial influence all the decision parameters. We then systematically removed regressors that did not reliably modulate the decision parameters to test whether the model fit was improved. Model comparisons were done according to widely applicable information criteria (WAIC), a Bayesian approach for estimating out-of-sample predictive accuracy with lower values indicating a better fit $[24,28]$. We used WAIC to approximate how well each model would perform on new participants. Thus, WAIC was computed across participants, where the log-likelihood for each participant's choice data were summed across trials (for the model fit of all the tested models, see Supplementary Information). We found that the full model with trial-to-trial affective modulators performed better than the base model without the influence of affective experience (WAIC $\mathrm{Base}_{\mathrm{B}}=2714$, WAIC $_{\text {Full }}=2702$ ). According to the full model, variations in valence did not reliably influenced any decision parameter, while arousal was positively associated with higher loss 
aversion, higher risk aversion, and higher choice-consistency (Figure S2 \& S3 in Supplementary Information). Next, we removed the valence modulators from the full model to see whether the model fit would improve, which resulted in a slightly lower information criterion $\left(\right.$ WAIC $\left._{\text {Adjusted }}=2698\right)$.

Each choice model was coded in Stan [20], fitted in R using the rstan package [21] with 8 sampling chains for 3,000 iterations each, 1,000 of which were discarded as warm-up samples $(16,000$ samples in total). Trace plots of the group level parameters were inspected for convergence and $\hat{R}$ values were ensured to be under 1.01 [26].

\section{Results}

\section{Affective experience model}

We investigated the influence of varying expectations, uncertainty, and prediction errors on affective experience using a computational model aimed at capturing momentary changes in subjective affective states [17]. The model was fit to valence and arousal data with hierarchical Bayesian estimation, which optimizes the tradeoff between random and fixedeffects models of individual differences. To summarize the model parameters, we plot the posterior distribution of the group level means (Figure 3) and report 95\% highest density intervals (HDIs) indicating the ranges, in which the most probable $95 \%$ of values fall.

The $95 \%$ HDI of $\boldsymbol{w}_{\boldsymbol{E} V}$ indicates that EV of the gambles had a positive impact on both valence $(95 \% \mathrm{HDI}=[0.21,0.28])$ and arousal $(95 \% \mathrm{HDI}=[0.24,0.32])$. Furthermore, $\boldsymbol{w}_{U}$ was negative for valence $(95 \% \mathrm{HDI}=[-0.1,-0.01])$ and positive for arousal $(95 \% \mathrm{HDI}=$ $[0.07,0.18])$, which indicates that uncertainty leads to negative valence and increased arousal. We also found that prediction errors had positive impact on both valence (95\% HDI $=[0.45,0.52])$ and arousal $(95 \% \mathrm{HDI}=[0.1,0.18])$. Interestingly, $w_{P E}$ was larger than $w_{E V}$ for the valence model indicating a stronger influence of prediction errors in comparison to the 
expected value. Finally, the posterior distribution of the forgetting factors showed lower values for valence $(95 \% \mathrm{HDI}=[0.18,0.31])$ in comparison to arousal $(95 \% \mathrm{HDI}=[0.32$, 0.55]), suggesting that the impact of earlier trials was stronger for arousal than for valence. Taken together, these results show that the affective impact of expected value, uncertainty of the expected value, and prediction errors are temporally integrated in momentary affective experience with differential impacts on valence and arousal.
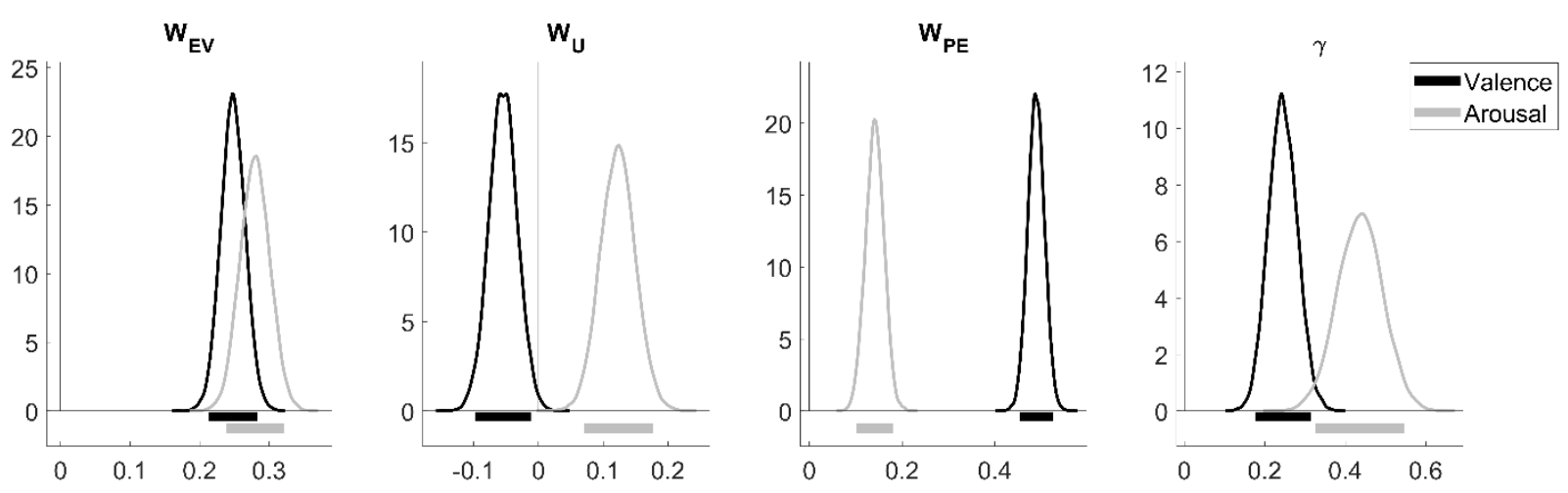

Figure 3 The posterior distributions on model parameter estimates showing the group level means of parameters of the valence (black) and arousal (grey) models. The peak value of each distribution represents the best estimate, while the width represents the uncertainty of the estimate. The marked ranges under the distributions represent $95 \%$ HDIs.

\section{Choice model}

We fit the choice model with and without the trial-by-trial affective modulations of decision variables (Figure S2 \& S3 in Supplementary Information). The model comparison showed that the model with affective modulation of decision parameters performed slightly better than the base model without the affective influence. Results showed that variations in valence did not reliably influenced any decision parameter, while the influence of arousal was 
reliably different from zero evidenced by the 95\% HDIs (Figure S3 in Supplementary Information). Finally, we removed the valence modulators from the full model, which resulted in a better model fit according to WAIC.

Here, we summarize the final adjusted model and report 95\% HDIs of the posterior distribution of the group level means (Figure 4). The posterior distribution of the group level parameters suggested that participants on average demonstrated risk averse behavior $(95 \%$ HDI on $\rho=[0.5,0.61])$. In addition, there was evidence against loss aversion as the $95 \%$ HDI on $\lambda$ included 1 (95\% HDI on $\lambda=[0.96,1.09])$. Finally, participants demonstrated choice consistency (95\% HDI on $c=[1.44,2.07])$. This model also included the impact of trial-bytrial arousal variations on the decision parameters. Interestingly, arousal was negatively associated with $\rho$ (95\% HDI on $\left.\beta_{A} \propto \rho=[-0.12,-0.03]\right)$ and positively associated with both $\lambda\left(95 \%\right.$ HDI on $\left.\beta_{A} \propto \lambda=[0.01,0.06]\right)$ and $c\left(95 \%\right.$ HDI on $\left.\beta_{A} \propto c=[0.01,0.54]\right)$. Taken together these estimates indicate that variations in experienced arousal influence risky decision-making by modulating loss aversion, risk sensitivity, and choice consistency. The findings suggest that increased arousal leads to a slightly increased loss averse and risk averse behavior as well as increased choice consistency in subsequent risky choices. 

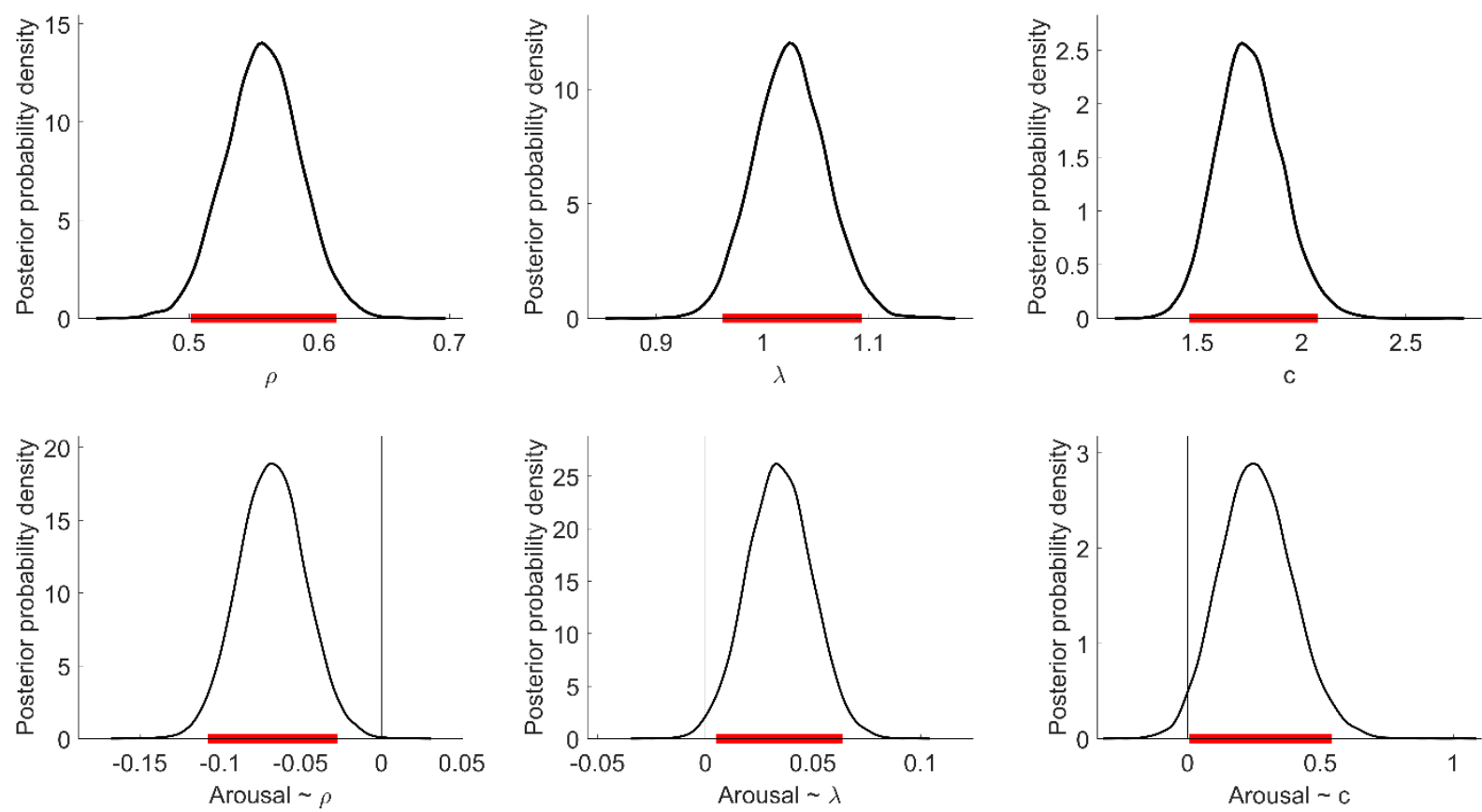

Figure 4 The posterior distributions on model parameter estimates showing the group level means and trial-to-trial influences of arousal on decision parameters. The upper panel shows the posterior distributions and the 95\% HDIs for group level parameters (marked ranges under the distributions). The lower panel shows the posterior distribution of the regression coefficients. The peak value of each distribution represents the best estimate, while the width represents the uncertainty of the estimate. 


\section{Discussion}

The current study set out to investigate how varying expectations, uncertainty, and prediction errors influence continuous affective experience, and how this dynamic representation of affect modulates ongoing subjective value computations during risky decision-making. Momentary affect was measured using self-reports of experienced valence and arousal. The results from the computational model of affect revealed that expected value, uncertainty, and prediction errors were temporally integrated into the overall affective experience with differential impact on valence and arousal features. Furthermore, we estimated the decision parameters allowing for them to change with affective experience prior to choice to assess whether subjective value computations are modulated as a function of changing affective experience. We found that experienced arousal prior to choice was associated with increased loss aversion and risk aversion, as well as higher choice consistency, whereas trial-by-trial valence did not reliably influence decision parameters. These findings present clear behavioral evidence for continuous affective modulation of subjective value computations during risky decision-making.

The posterior distribution of parameter estimates of the computational affect model indicated that both expected value and prediction errors were positively associated with valence and arousal. Previous investigations using the same computational model found that moment-to-moment happiness ratings were partly shaped by expected value and prediction error, with significantly stronger impact of the latter $[17,25]$. The current evidence on the impact of expected value and prediction error on valence ratings are in line with these previous findings. Moreover, our results indicate that it is possible to use a similar model to study moment-to-moment arousal as a function of previously encountered events. Critically, we also have shown that uncertainty of the expected value can also be integrated in the same model. Importantly, uncertainty was associated with negative affect and increased arousal, 
which is in line with previous findings showing that uncertainty can cause unpleasant affect $[16,29]$ and anxiety [30]. Furthermore, previous research suggests that uncertainty signals from the environment drive the physiological arousal systems [31, 32]. We additionally found that group level forgetting factor was larger in arousal relative to valence, which indicates that the affective impact of previous trials is stronger on arousal in comparison to valence. This also means that valence fluctuates more closely with the current stimuli compared to arousal, which is in agreement with previous findings from studies with affective images [16]. Taken together, the temporally sensitive modeling approach we adopted proves a useful strategy for revealing the differential dynamics of valence and arousal features of affective experience depending on varying expectations, uncertainty, and prediction errors during risky decision-making.

The computational modeling of choice behavior revealed that allowing the decision parameters to change with affective experience improved the model fit. This suggests that subjective value calculations are modulated by trial-by-trial variations in affective experience. We found that arousal, but not valence, reported in the previous trial was associated with increased loss aversion, risk aversion, and choice consistency for the subsequent decision. Previous research has attributed loss aversive behavior at an individual level to higher arousal responses and increased amygdala activations to losses relative to gains $[13,33]$. The current results support these earlier findings and critically extend them by showing the continuous modulation of decision parameters by the affective impact of previous gains and losses. Recent findings also indicate that previous events influence subsequent risky decision-making $[34,35]$. Positive previous outcomes in risky monetary decisions can increase loss aversion and choice consistency for subsequent risky choices [34]. These contextual influences suggest that risky decision-making is fundamentally dynamic and temporally dependent on the impact of previous events. Affect, reflecting the individual's 
ongoing relationship with its surroundings, represents the cumulative impact of previous events $[14,15]$. Hence, it is a critical mechanism through which recent events influence subsequent behavior. The effects we identified provide evidence for the underlying affective correlates of the impact of recent events on subsequent risky choices.

Moreover, the changes in uncertainty due to changes in the environmental signals seem to drive arousal [32]. Previous investigations show that the extent of the correlation between uncertainty and arousal predicts individual performance in a probabilistic learning task [31]. Moreover, decision uncertainty in a perceptual decision task may lead to rapid changes in pupil-linked arousal, which in turn shapes the choice behavior in subsequent trials [36]. Additionally, in risky choice, arousal is associated with anticipation of risks [10, 11]. Hence, these earlier findings together with the current results suggest that arousal represents the changes in uncertainty signals from the environment and modulates ongoing behavior (see also, [37]). Here, our findings indicate that subjective arousal encodes varying expectations, uncertainty, and predictions errors and influences subsequent risky choice by modulating ongoing subjective value computations.

We find that there are reliable effects of previous affective experience on various decision parameters. It is, however, critical to note that affect may also modulate risky decision-making through other mechanisms. For instance, anticipated affective states and emotions may also influence subjective value of the choice options [38, 39]. Moreover, other decision parameters such as probability weighting, not modeled in our study, may also be influenced by variations in affective experience [40, 41]. The further development of temporally and contextually sensitive models including neural and physiological correlates will be critical in understanding the continuous modulatory influence of affective processing on decision-making under risk. 
The brain encodes the individual's beliefs about the hidden states of the world [42]. These beliefs are probability distributions representing expectations and uncertainty. Hence, decision-making is about sampling the most appropriate action based on expected rewards and losses as well as the uncertainty around these expectations [43]. The current study provides clear behavioral evidence that ongoing affective experience encodes varying expectations and uncertainty that underlie our decisions, and it keeps track of the recent prediction error history. We have also shown that the arousal feature of this dynamic representation of affective experience, then, continuously modulates risky decision-making. Taken together, these findings point towards the benefit of adopting an experimental framework that attempts to understand the dynamic aspects of the involvement of affect in decision-making to uncover the mechanisms through which affect modulates our choices.

\section{References}

1. Phelps, E. A., Lempert, K. M., \& Sokol-Hessner, P. Emotion and decision-making: Multiple modulatory neural circuits. Annual Review of Neuroscience 37, 263-287 (2014).

2. Lerner, J. S., Li, Y., Valdesolo, P., \& Kassam, K. S. Emotion and decision making. Annual Review Psychology 66, 799-823 (2015).

3. Sokol-Hessner, P. \& Phelps, E. A. Affect, decision-making, and value: Neural and psychological mechanism in Handbook of Value (ed. Brosch, T. \& Sander, D.) 197-222 (Oxford University Press, 2016).

4. Lerner, J. S. \& Keltner, D. Fear, anger, and risk. Journal of Personality and Social Psychology 81, 146-159 (2001).

5. Baumann, J. \& DeSteno, D. Context explains divergent effects of anger in risk taking. Emotion 12, 1196-1199 (2012). 
6. Kugler, T., Connolly, T., \& Ordóñez, L. D. Emotion, decision, and risk: Betting on gambles versus betting on people. Journal of Behavioral Decision Making 25, 123-134 (2012).

7. Ferrer, R.A., Maclay, A., Litvak P. M., \& Lerner J. S. Revisiting the effects of anger on risk-taking: Empirical and meta-analytic evidence for differences between males and females. Journal of Behavioral Decision Making 30, 516-526 (2017).

8. Ferrer, R. A. \& Ellis, E. M. Preliminary evidence for differential effects of integral and incidental emotions on risk perception and behavioral intentions: A meta-analysis of eight experiments. Journal of Behavioral Decision Making 34, 275-289 (2021).

9. Västfjäll, D. et al. The arithmetic of emotion: Integration of incidental and integral affect in judgments and decisions. Frontiers in Psychology 7, 325 (2016).

10. Bechara A., Damasio H., Tranel D., \& Damasio A. R. Deciding advantageously before knowing the advantageous strategy. Science 275, 1293-1295 (1997).

11. FeldmanHall, O., Glimcher, P., Baker, A. G., \& Phelps E. A. Emotion and decisionmaking under uncertainty: physiological arousal predicts increased gambling during ambiguity but not risk. Journal of Experimental Psychology: General 145, 1255-1262 (2016).

12. Kahneman, D., \& Tversky, A. Prospect theory: An analysis of decision under risk. Econometrica 47, 263-291 (1979).

13. Sokol-Hessner, P. et al. Thinking like a trader selectively reduces individuals' loss aversion. PNAS 106, 5035-5040 (2009).

14. Barrett L. F. The theory of constructed emotion: an active inference account of interoception and categorization. Social Cognitive and Affective Neuroscience 12, 1-23 (2017). 
15. Asutay E. et al. Affective calculus: The construction of affect through averaging information over time. Emotion 21, 159-174 (2021).

16. Asutay E., Genevsky, A., Hamilton, P., \& Västfjäll, D. Affective context and uncertainty drive momentary affective experience. Emotion. Advance online publication (2020).

17. Rutledge, R. B., Skandali, N., Dayan, P., \& Dolan, R. J. A computational and neural model of momentary subjective well-being. PNAS 111, 12252-12257 (2014).

18. Huk, A., Bonnen, K., \& He, B. J. Beyond trial-based paradigms: Continuous behavior, ongoing neural activity, and natural stimuli. Journal of Neuroscience 38, 7551-7558 (2018).

19. Hutchinson, J. B. \& Barrett, L. F. The power of predictions: An emerging paradigm for psychological research. Current Directions in Psychological Science 28, 280-291 (2019).

20. Carpenter, B. et al. Stan: A probabilistic programming language. Journal of Statistical Software 76 (2017).

21. Stan Development Team. RStan: The R interface to Stan. http://mc-stan.org (2018).

22. Kruschke, J. K. Doing Bayesian data analysis. 2nd ed. (Academic Press, 2015).

23. Lee, M. D., \& Wagenmakers, E.-J. Bayesian cognitive modeling. (Cambridge University Press, 2013).

24. Gelman A. et al. Bayesian Data Analysis. 3rd ed. (Chapman and Hall/CRC, 2014).

25. Vanhasbroeck, N. et al. Testing a computational model of subjective well-being: a preregistered replication of Rutledge et al. (2014). Cognition and Emotion. (2021).

26. Gelman, A., \& Rubin, D. B. Inference from iterative simulation using multiple sequences. Statistical Science 7, 457-511 (1992).

27. Frank M.J. et al. fMRI and EEG predictors of dynamic decision parameters during human reinforcement learning. The Journal of Neuroscience 35, 485-494 (2015). 
28. Watanabe, S. Asymptotic equivalence of Bayes cross validation and widely applicable information criterion in singular learning theory. Journal of Machine Learning Research 11, 3571-3594 (2010).

29. Joffily, M \& Coricelli, G. Emotional valence and the free energy principle. PLoS Computational Biology 9, e1003094 (2013).

30. Herry, C. et al. Processing of temporal unpredictability in human and animal amygdala. The Journal of Neuroscience 38, 7551-7558 (2018).

31. de Berger et al. Computations of uncertainty mediate acute stress responses in humans. Nature Communications 7, 10996 (2016).

32. Yu, A. J. \& Dayan, P. Uncertainty, neuromodulation, and attention. Neuron 46, 681-692 (2005).

33. Sokol-Hessner P., Camerer C. F., \& Phelps E. A. Emotion regulation reduces loss aversion and decreases amygdala responses to losses. Social Cognitive Affective Neuroscience 8, 341-350 (2012).

34. Brooks, H. R. \& Sokol-Hessner, P. Quantifying the immediate computational effects of preceding outcomes on subsequent risky choices. Scientific Reports 10, 9878 (2020).

35. Juechems, K. Balaguer, J., Ruz, M., \& Summerfield, C. Ventromedial prefrontal cortex encodes a latent estimate of cumulative reward. Neuron 93, 705-714 (2017).

36. Urai, A., Braun, A. \& Donner, T. Pupil-linked arousal is driven by decision uncertainty and alters serial choice bias. Nature Communications 8, 14637 (2017).

37. Ashton-Jones, G. \& Cohen, J. D. An integrative theory of Locus CoeruleusNorepinephrine function: adaptive gain and optimal performance. Annual Reviews of Neuroscience 28, 403-450 (2005).

38. Mellers, B. A., Yin, S., \& Berman, J. Z. Reconciling loss aversion and gain seeking in judged emotions. Current Directions in Psychological Science 30, 95-102 (2021). 
39. Sokol-Hessner, P. \& Rutledge, R. B. The psychological and neural basis of loss aversion. Current Directions in Psychological Science 28, 20-27 (2019).

40. Hsee, C. K. \& Rottenstreich, Y. Music, pandas, and muggers: on the affective psychology of value. Journal of Experimental Psychology: General 133, 23-30 (2004).

41. Västfjäll, D., \& Slovic, P. Cognition and emotion in judgment and decision making. in Handbook of Cognition and Emotion (eds. Robinson, MD, Watkins, ER., \& HarmonJones, E.) 252-271 (Guilford Press, 2013).

42. Friston, K. The free-energy principle: a unified brain theory? Nature Reviews Neuroscience 11, 70-87 (2010).

43. Bach, D. R. \& Dolan, R. J. Knowing how much you don't know: a neural organization of uncertainty estimates. Nature Reviews Neuroscience 13, 572-586 (2012).

\section{Author contributions}

EA and DV developed the study concept and design. EA conducted data collection and analysis and wrote the manuscript. DV provided critical revisions. Both authors approved the final version of the manuscript for submission.

\section{Competing interests}

The authors declare no competing interests

\section{Data availability}

All data and modeling codes are available at https://osf.io/ryfu9/. 


\section{Supplementary Files}

This is a list of supplementary files associated with this preprint. Click to download.

- SOM.docx 